\title{
CP VIOLATION IN SUPERSYMMETRIC SEESAW MODELS
}

\author{
JUNJI HISANO \\ ICRR, University of Tokyo \\ 5-1-5 Kashiwa-no-Ha \\ Kashiwa, 277-8582, Japan
}

\begin{abstract}
In supersymmetric (SUSY) extensions of the seesaw mechanism the neutrino Yukawa interaction induces the flavor and $\mathrm{CP}$ violating sfermion mass terms via the radiative correction. In this article we review the $\mathrm{CP}$ violating phenomena in the SUSY seesaw models.
\end{abstract}

\section{Introduction}

The seesaw mechanism ${ }^{1}$, which explains tiny neutrino masses, is one of the most promising models beyond the standard model (SM), after discovery of the neutrino oscillation. It is also compatible with the matter unification in the GUTs. In the SO(10) GUT quarks and leptons may be embedded into 16 dimensional multiplets for each generation, in cooperation with the right-handed neutrinos. In addition to it, the baryon number in the universe may be explained by the leptogenesis ${ }^{2}$. Hence, it is important to search for signals in the models.

Supersymmetry (SUSY) is expected in the seesaw mechanism so that the hierarchical structure is stabilized. If the SUSY breaking terms in the minimal SUSY SM (MSSM) comes from dynamics whose energy scale is above that of the seesaw mechanism or the extension, the neutrino Yukawa interaction generates the flavor and CP violating SUSY breaking terms ${ }^{34}$. Thus, the flavor and CP violating phenomena are sensitive to the SUSY seesaw models.

This paper is concentrated into the CP violating aspects in the SUSY seesaw model and the extension to the SUSY GUT. The lepton flavor violating (LFV) processes and the related topics in the models are reviewed by Masiero in this volume. 


\section{Minimal SUSY seesaw model}

In the minimal SUSY seesaw model, in which only three right-handed neutrinos are introduced, the SUSY breaking terms in the lepton sector are sensitive to the neutrino Yukawa coupling. Thus, the CP violation in the minimal SUSY seesaw model may lead to the T-odd asymmetry in the lepton-flavor violating lepton decay, such as $\mu \rightarrow 3 e$, and the leptonic EDMs. In this section, we review the flavor structure in the leptonic SUSY breaking terms, which is predicted in the model, and the $\mathrm{CP}$ violating observables.

In the minimal SUSY seesaw model the relevant leptonic part of the superpotential is

$$
W_{\text {seesaw }}=f_{i j}^{\nu} L_{i} \bar{N}_{j} \bar{H}_{f}+f_{i j}^{l} \bar{E}_{i} L_{j} H_{f}+\frac{1}{2} M_{i j} \bar{N}_{i} \bar{N}_{j}
$$

where the indexes $i, j$ run over three generations and $M_{i j}$ is the heavy singlet neutrino mass matrix. In addition to the three charged lepton masses, this superpotential has eighteen physical parameters, including six real mixing angles and six CP-violating phases, because the Yukawa coupling and the Majorana mass matrices are given after removing unphysical phases as $f_{i j}^{l}=$ $f_{l_{i}} \delta_{i j}, f_{i j}^{\nu}=X_{i k}^{\star} f_{\nu_{k}} \mathrm{e}^{-i \varphi_{\nu_{k}}} W_{k j}^{\star} \mathrm{e}^{-i \bar{\varphi}_{\nu_{k}}}$, and $M_{i j}=\delta_{i j} M_{N_{k}}$. Here, $\sum_{i} \varphi_{\nu_{i}}=0$ and $\sum_{i} \bar{\varphi}_{\nu_{i}}=0$, and each $W$ and $X$ are unitary matrices with one phase.

At low energies the effective theory after integrating out the righthanded neutrinos is given by the effective superpotential,

$$
W_{\text {eff }}=f_{l_{i}} \bar{E}_{i} L_{i} H_{f}+\frac{1}{2 v^{2} \sin ^{2} \beta}\left(m_{\nu}\right)_{i j}\left(L_{i} \bar{H}_{f}\right)\left(L_{j} \bar{H}_{f}\right),
$$

where we work in a basis in which the charged lepton Yukawa couplings are diagonal. The second term in (2) leads to the light neutrino masses and mixings. The explicit form of the small neutrino mass matrix $\left(m_{\nu}\right)$ is given by $\left(m_{\nu}\right)_{i j}=\sum_{k} f_{i k}^{\nu} f_{j k}^{\nu} v^{2} \sin ^{2} \beta / M_{N_{k}}$. The light neutrino mass matrix $\left(m_{\nu}\right)$ is symmetric, with nine parameters, including three real mixing angles and three CP-violating phases. It can be diagonalized by a unitary matrix $Z$ as $Z^{T} m_{\nu} Z=m_{\nu}^{D}$. By redefinition of fields one can rewrite $Z \equiv U P$, where $P \equiv \operatorname{diag}\left(e^{i \phi_{1}}, e^{i \phi_{2}}, 1\right)$ and $U$ is the MNS matrix, with the three real mixing angles and the remaining $\mathrm{CP}$-violating phase.

The abilities to probe the minimal seesaw model by the low-energy neutrino experiments, such as the neutrino oscillation and the double $\beta$ decay, are limited. Nine parameters associated with the heavy-neutrino sector cannot be measured in a direct way. However, we may probe the model by phenomena induced by the SUSY breaking terms in the MSSM. 
If the SUSY-breaking terms are generated above the right-handed neutrino mass scale, the renormalization effects may induce sizable LFV slepton mass terms, which lead to the LFV charged lepton decays. If the SUSYbreaking parameters at the GUT scale or the Planck scale are universal, off-diagonal components in the left-handed slepton mass matrix $\left(m_{\tilde{l}_{L}}^{2}\right)$ and the trilinear slepton coupling $\left(A_{l}\right)$ take the approximate forms,

$$
\begin{aligned}
\left(m_{\tilde{l}_{L}}^{2}\right)_{i j} & \simeq-\sum_{k} \frac{f_{i k}^{\nu \star} f_{j k}^{\nu}}{16 \pi^{2}}\left[m_{0}^{2}\left(3 \log \frac{M_{G}^{2}}{M_{N_{k}}^{2}}+1\right)+A_{0}^{2}\left(\log \frac{M_{G}^{2}}{M_{N_{k}}^{2}}+1\right)\right], \\
\left(A_{l}\right)_{i j} & \simeq-\sum_{k} f_{l_{i}} A_{0} \frac{f_{i k}^{\nu \star} f_{j k}^{\nu}}{16 \pi^{2}}\left[\log \frac{M_{G}^{2}}{M_{N_{k}}^{2}}+1\right],
\end{aligned}
$$

where $i \neq j$, and the off-diagonal components of the right-handed slepton mass matrix are suppressed. Here, we include the one-loop finite parts ${ }^{5}$ in the radiative correction, and ignore terms of higher order in $f_{l}$, assuming that $\tan \beta$ is not extremely large.

The non-vanishing off-diagonal components in $\left(m_{\tilde{l}_{L}}^{2}\right)$ and $\left(A_{l}\right)$ predict the charged lepton-flavor violating decays, whose measurements supply the information about the seesaw model, which is independent of the low-energy neutrino experiments. Ignoring the one-loop finite corrections, the terms in Eq. (3) are proportional to $H_{i j}=\sum_{k} f_{i k}^{\nu \star} f_{j k}^{\nu} \log \left(M_{G}^{2} / M_{N_{k}}^{2}\right)$. Here, the Hermitian matrix $H$, whose diagonal terms are real and positive, is defined in terms of $f^{\nu}$ and the heavy neutrino masses $M_{N_{k}}$. This matrix has nine parameters including three phases, which are clearly independent of the parameters in $\left(m_{\nu}\right)$. Thus two matrices $\left(m_{\nu}\right)$ and $H$ together provide the required eighteen parameters, including six $\mathrm{CP}$-violating phases, by which we can parameterize the minimal SUSY seesaw model ${ }^{6}$. The off-diagonal terms, $H_{i j}(i \neq j)$, are related to the LFV $l_{i}-l_{j}$ transition, and they are related to the LFV charged lepton decays.

Now we discuss the CP violation in the minimal SUSY seesaw model. The CP violating observables of the charged leptons are the T-odd asymmetry in $l \rightarrow 3 l^{\prime}$ and the leptonic EDMs.

First, we discuss the T-odd asymmetry in $\mu^{+} \rightarrow e^{+} e^{-} e^{+}$. In order for $\mathrm{CP}$ violation to appear in any process, interference between different terms in the amplitude for the process must occur. Therefore, all possible observables in $l \rightarrow l^{\prime} \gamma$ decays, such as differences between the $\mu^{+} \rightarrow e^{+} \gamma$ and $\mu^{-} \rightarrow e^{-} \gamma$ rates, vanish in the leading order of perturbation theory. Moreover, the process $\mu^{-} \rightarrow e^{-} \gamma$ is not measurable with high accuracy because of the large backgrounds. However, when muons are polarized, a 
T-odd asymmetry for final-state particles in $\mu^{+} \rightarrow e^{+} e^{+} e^{-}$can be defined. Since CPT is conserved, the T-odd asymmetry measures the amount of CP violation in the model.

The muon polarization vector $\vec{P}$ can be defined in the coordinate system in which the $z$ axis is taken to be the direction of the electron momentum, the $x$ axis the direction of the most energetic positron momentum, and the $(z \times x)$ plane defines the decay plane perpendicular to the $y$ axis. The T-odd asymmetry is then defined ${ }^{7}$ by

$$
A_{T}=\frac{N\left(P_{y}>0\right)-N\left(P_{y}<0\right)}{N\left(P_{y}>0\right)+N\left(P_{y}<0\right)}
$$

where $N\left(P_{i}>(<) 0\right)$ denotes the number of events with a positive (negative) $P_{i}$ component for the muon polarization. $A_{T}$ is limited to be below $24 \%^{8}$.

In the branching ratio for $\mu \rightarrow 3 e$, the contribution from photonic penguin diagram tends to dominate due to the phase-space integral while the $Z$ penguin and box diagrams also gives the contribution. Then, assuming that the photonic penguin diagram dominates in $\operatorname{Br}\left(\mu^{+} \rightarrow e^{+} e^{+} e^{-}\right)$, the T-odd asymmetry $A_{T}$, induced by non-vanishing off-diagonal terms in $\left(m_{L}^{2}\right)$, is approximately given ${ }^{9}$ by

$$
\begin{aligned}
A_{T}= & \frac{\operatorname{Im}\left[\left(\Delta_{12}^{l}\right)_{L}\left(\Delta_{23}^{l}\right)_{L}\left(\Delta_{31}^{l}\right)_{L}\right]}{\left|\left(\Delta_{12}^{l}\right)_{L}\right|^{2}} \\
& \times \frac{0.039+0.196 \tan \beta+0.017 / \tan \beta}{\left|(1+2.4 \tan \beta)-\frac{\left(\Delta_{23}^{l}\right)_{L}\left(\Delta_{31}^{l}\right)_{L}}{\left(\Delta_{21}^{l}\right)_{L}}(0.64+1.12 \tan \beta)\right|^{2}},
\end{aligned}
$$

where $\left(\Delta_{i j}^{l}\right)_{L} \equiv\left(\left(m_{L}^{2}\right)_{i j} / m_{S}^{2}\right)$. Here, in writing Eq. (5), we have taken $\left(A_{l}\right)_{i j}=0(i \neq j)$, for simplicity. We see explicitly how $A_{T}$ in Eq. (5) depends on the Jarlskog invariant $J_{L}=\operatorname{Im}\left[\left(m_{\tilde{l}_{L}}^{2}\right)_{12}\left(m_{\tilde{l}_{L}}^{2}\right)_{23}\left(m_{\tilde{l}_{L}}^{2}\right)_{31}\right]$. It is found that $A_{T}$ could in principle reach $\sim 10 \%$. However, if $\operatorname{Im}\left[\left(\Delta_{12}^{l}\right)_{L}\left(\Delta_{23}^{l}\right)_{L}\left(\Delta_{31}^{l}\right)_{L}\right] \ll\left|\left(\Delta_{12}^{l}\right)_{L}\right|^{2}$, as one might expect, or if $\tan \beta \gg 1$, $A_{T}$ is suppressed.

In Fig. 1 we show (a) the branching ratios for the decays $\mu \rightarrow e \gamma$ and $\mu \rightarrow 3 e$ and (b) the T-odd asymmetry $A_{T}$ in $\mu^{+} \rightarrow e^{+} e^{+} e^{-}$decay, as functions of the common soft mass $m_{0}$. For the neutrino parameters and others in the minimal SUSY seesaw model, see Ref. ${ }^{9}$. When the branching ratio for $\mu \rightarrow e \gamma$ is suppressed due to the accidental cancellation, the Todd asymmetry is enhanced. This is because the penguin contribution is suppressed and and becomes comparable to the other contributions. 

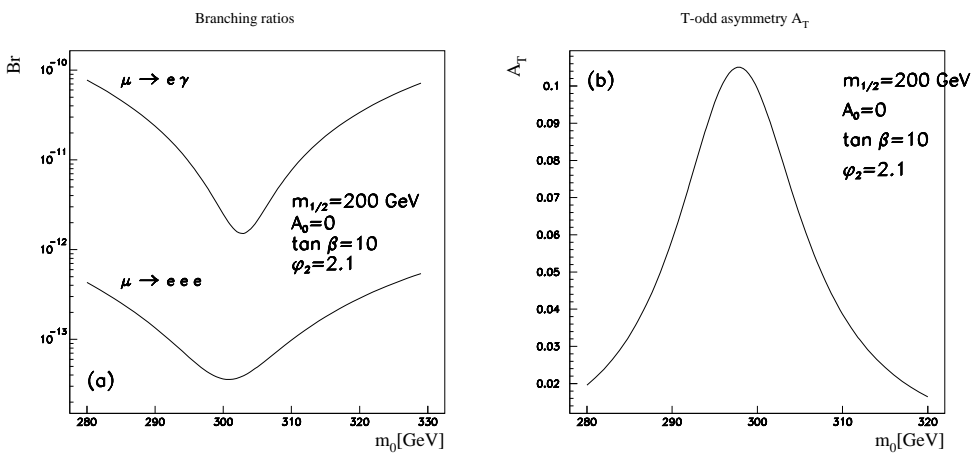

Figure 1. (a) Branching ratios for the decays $\mu \rightarrow e \gamma$ and $\mu \rightarrow 3 e$ and (b) the T-odd asymmetry $A_{T}$ in $\mu^{+} \rightarrow e^{+} e^{+} e^{-}$decay, as functions of the common soft mass $m_{0}$.

The leptonic EDMs depend on the flavor-diagonal Jarlskog invariants. If the source of the flavor violation comes from only the Yukawa interactions in the minimal SUSY seesaw model, the flavor-diagonal Jarlskog invariants, which consist of the Yukawa coupling constants, are given as

$$
J_{\text {edm }}^{(i)}=\operatorname{Im}\left[f^{l \star}\left[f^{\nu} f^{\nu \dagger}\left[f^{l T} f^{l \star}, f^{\nu} f^{\nu \dagger}\right] f^{\nu} f^{\nu \dagger}\right]_{i i}\right] .
$$

These are much suppressed by the Yukawa coupling constants. This situation is similar to the hadronic EDMs in the SM, where the CP violation comes from the CKM matrix. However, in the minimal SUSY seesaw model, when the right-handed neutrinos are not degenerate in mass, we have other flavor-diagonal Jarlskog invariants,

$$
J_{\text {edm }}^{(i) \prime}=\operatorname{Im}\left[f^{l \star}\left[f^{\nu} f^{\nu \dagger}, f^{\nu} \log \frac{M_{G}^{2}}{M_{N}^{2}} f^{\nu \dagger}\right]_{i i}\right] .
$$

Obviously, they vanish when the right-handed neutrino masses are degenerate. Thus, it is expected that the leptonic EDMs are enhanced significantly when the right-handed neutrinos masses are not degenerate ${ }^{10}$.

When the SUSY breaking terms are generated as in Eq. (3), the nontrivial Jarlskog invariants, $J_{\text {edm }}^{(i) \prime}$, are given as $^{5}$

$$
\operatorname{Im}\left[\left[\left(A_{l}\right)\left(m_{\tilde{l}_{L}}^{2}\right)\right]_{i i}\right]=\frac{A_{0} m_{0}^{2}}{(4 \pi)^{4}} J_{\mathrm{edm}}^{(i) \prime},
$$

and the leptonic EDMs are proportional to them. It is shown that the electron and muon EDMs may reach to $10^{-29}$ and $10^{-27} \mathrm{ecm}$, respectively, for $m_{S} \sim 200 \mathrm{GeV}^{5}$. The $\mathrm{CP}$ violating phases contributing to the leptonic 
EDMs are independent of those in other $\mathrm{CP}$ violating observables, such as the T-odd asymmetry in $\mu \rightarrow 3 e$ or the neutrino oscillation.

\section{SUSY SU(5) GUT with right-handed neutrinos}

When the SUSY seesaw model is extended to the SUSY GUTs, the neutrino Yukawa interaction also induces the flavor violation in the hadronic sector via the radiative corrections to the squark SUSY breaking terms. In this section, we discuss the hadronic CP violation in the SUSY GUTs with the right-handed neutrinos. Here, we take the SUSY SU(5) GUT for simplicity. In this model, doublet leptons and right-handed down-type quarks are embedded in common 5-dimensional multiplets, while doublet quarks, right-handed up-type quarks, and right-handed charged leptons are in the 10-dimensional ones. Thus, the neutrino Yukawa coupling induces the right-handed down-type squark mixing, and this leads to rich flavor and $\mathrm{CP}$ violating phenomena in hadrons. They are also correlated due to the GUT relation, especially processes of the second and third generation transition ${ }^{11}$.

First, we review the flavor structure in the squark and slepton mass matrices in the SUSY SU(5) GUT with the right-handed neutrinos. The Yukawa interactions for quarks and leptons and the Majorana mass terms for the right-handed neutrinos in this model are given by the following superpotential,

$$
W=\frac{1}{4} f_{i j}^{u} \Psi_{i} \Psi_{j} H+\sqrt{2} f_{i j}^{d} \Psi_{i} \Phi_{j} \bar{H}+f_{i j}^{\nu} \Phi_{i} \bar{N}_{j} H+M_{i j} \bar{N}_{i} \bar{N}_{j},
$$

where $\Psi$ and $\Phi$ are for $\mathbf{1 0}$ - and $\overline{\mathbf{5}}$-dimensional multiplets, respectively, and $\bar{N}$ is for the right-handed neutrinos. $H(\bar{H})$ is $\mathbf{5}-(\overline{\mathbf{5}}-)$ dimensional Higgs multiplets. After removing the unphysical degrees of freedom, the Yukawa coupling constants in Eq. (9) are given as $f_{i j}^{u}=V_{k i} f_{u_{k}} \mathrm{e}^{i \varphi_{u_{k}} V_{k j}}, f_{i j}^{d}=f_{d_{i}} \delta_{i j}$, and $f_{i j}^{\nu}=\mathrm{e}^{i \varphi_{d_{i}}} U_{i j}^{\star} f_{\nu_{j}}$. Here, $\varphi_{u}$ and $\varphi_{d}$ are CP-violating phases inherent in the SUSY SU(5) GUT. They satisfy $\sum_{i} \varphi_{f_{i}}=0(f=u$ and $d)$. The unitary matrix $V$ is the CKM matrix in the extension of the SM to the SUSY SU(5) GUT, and each unitary matrices $U$ and $V$ have only a phase. When the Majorana mass matrix for the right-handed neutrinos is diagonal, $U$ is the MNS matrix observed in the neutrino oscillation. In this section we assume the diagonal Majorana mass matrix in order to avoid the complexity due to the structure.

The colored Higgs multiplets $H_{c}$ and $\bar{H}_{c}$ are introduced in $H$ and $\bar{H}$ as $\mathrm{SU}(5)$ partners of the Higgs doublets in the MSSM, respectively. They have 
new flavor-violating interactions in Eq. (9). If the SUSY-breaking terms in the MSSM are generated by dynamics above the colored Higgs masses, such as in the gravity mediation, the sfermion mass terms may get sizable corrections by the colored Higgs interactions. In the minimal supergravity scenario the SUSY breaking terms are supposed to be given at the reduced Planck mass scale $\left(M_{G}\right)$. In this case, the flavor-violating SUSY breaking mass terms at low energy are induced by the radiative correction, and they are qualitatively given in a flavor basis as

$$
\begin{aligned}
& \left(m_{\tilde{u}_{L}}^{2}\right)_{i j} \simeq-V_{i 3} V_{j 3}^{\star} \frac{f_{b}^{2}}{(4 \pi)^{2}}\left(3 m_{0}^{2}+A_{0}^{2}\right)\left(2 \log \frac{M_{G}^{2}}{M_{H_{c}}^{2}}+\log \frac{M_{H_{c}}^{2}}{m_{S}^{2}}\right), \\
& \left(m_{\tilde{u}_{R}}^{2}\right)_{i j} \simeq-\mathrm{e}^{-i \varphi_{u_{i j} j}} V_{i 3}^{\star} V_{j 3} \frac{2 f_{b}^{2}}{(4 \pi)^{2}}\left(3 m_{0}^{2}+A_{0}^{2}\right) \log \frac{M_{G}^{2}}{M_{H_{c}}^{2}}, \\
& \left(m_{\tilde{d}_{L}}^{2}\right)_{i j} \simeq-V_{3 i}^{\star} V_{3 j} \frac{f_{t}^{2}}{(4 \pi)^{2}}\left(3 m_{0}^{2}+A_{0}^{2}\right) \quad\left(3 \log \frac{M_{G}^{2}}{M_{H_{c}}^{2}}+\log \frac{M_{H_{c}}^{2}}{m_{S}^{2}}\right), \\
& \left(m_{\tilde{d}_{R}}^{2}\right)_{i j} \simeq-\mathrm{e}^{i \varphi_{d_{i j}}} U_{i k}^{\star} U_{j k} \frac{f_{\nu_{k}}^{2}}{(4 \pi)^{2}}\left(3 m_{0}^{2}+A_{0}^{2}\right) \log \frac{M_{G}^{2}}{M_{H_{c}}^{2}}, \\
& \left(m_{\tilde{l}_{L}}^{2}\right)_{i j} \simeq-U_{i k} U_{j k}^{\star} \frac{f_{\nu_{k}}^{2}}{(4 \pi)^{2}}\left(3 m_{0}^{2}+A_{0}^{2}\right) \log \frac{M_{G}^{2}}{M_{N_{k}}^{2}}, \\
& \left(m_{\tilde{e}_{R}}^{2}\right)_{i j} \simeq-\mathrm{e}^{i \varphi_{d_{i j}}} V_{3 i} V_{3 j}^{\star} \frac{3 f_{t}^{2}}{(4 \pi)^{2}}\left(3 m_{0}^{2}+A_{0}^{2}\right) \quad \log \frac{M_{G}^{2}}{M_{H_{c}}^{2}},
\end{aligned}
$$

with $i \neq j$, where $\varphi_{u_{i j}} \equiv \varphi_{u_{i}}-\varphi_{u_{j}}$ and $\varphi_{d_{i j}} \equiv \varphi_{d_{i}}-\varphi_{d_{i}}$ and $M_{H_{c}}$ is the colored Higgs mass. $f_{t}$ is the top quark Yukawa coupling constant while $f_{b}$ is for the bottom quark. As mentioned above, the off-diagonal components in the right-handed squarks and slepton mass matrices are induced by the colored Higgs interactions, and they depend on the CP-violating phases in the SUSY SU(5) GUT with the right-handed neutrinos ${ }^{12}$.

When both the left-handed and right-handed squarks have the offdiagonal components in the mass matrices, the EDMs and CEDMs for the light quarks are enhanced significantly by the heavier quark mass ${ }^{1314}$. The EDMs and CEDMs contribute to the hadronic EDMs, and they are constrained by the observations. In the SUSY SU(5) GUT with the right-handed neutrinos, the neutrino Yukawa coupling induces the flavorviolating mass terms for the right-handed down-type squarks. The flavorviolating mass terms for the left-handed down-type squarks are expected to be dominated by the radiative correction, which is controlled by the CKM matrix, induced by the top quark Yukawa coupling as in Eq. (10). Then, we can investigate or constrain the structure in the neutrino sector by the 
hadronic EDMs, which is generated by the CEDMs and EDMs of the down and strange quarks. ${ }^{\mathrm{a}}$

The CEDMs for the light quarks, including the strange quark, contribute to the hadronic EDMs since the CP-violating nucleon coupling is induced by them. Here, we consider only the CEDMs for the light quarks. While the EDMs for the up and down quarks contribute to the neutron EDM, it is found that the EDM contributions are comparable to or smaller than the CEDM contributions.

The CEDMs of the down-type light quarks derived by the flavor violation in the both the left-handed and right-handed quark mass matrices are given by the following dominant contribution, which is enhanced by the heavier quark masses,

$$
d_{d_{i}}^{C}=\frac{\alpha_{s}}{4 \pi} \frac{m_{\tilde{g}}}{\bar{m}_{\tilde{d}}^{2}} f\left(\frac{m_{\tilde{g}}^{2}}{\bar{m}_{\tilde{d}}^{2}}\right) \sum_{j} \operatorname{Im}\left[\left(\Delta_{i j}^{d}\right)_{L}\left(\Delta_{j}^{d}\right)_{L R}\left(\Delta_{j i}^{d}\right)_{R}\right],
$$

where $m_{\tilde{g}}$ and $\bar{m}_{\tilde{d}}$ are the gluino and averaged squark masses. The mass insertion parameters are defined as $\left(\Delta_{i j}^{d}\right)_{L / R} \equiv\left(m_{\tilde{d}_{L / R}}^{2}\right)_{i j} / \bar{m}_{\tilde{f}}^{2}$ and $\left(\Delta_{i}^{d}\right)_{L R} \equiv m_{d_{i}}\left(A_{i}^{(d)}-\mu \tan \beta\right) / \bar{m}_{\tilde{d}}^{2}$. The function $f(x)$ is given in Ref. 16 and $f(1)=-11 / 180$.

In order to translate the CEDMs of the light quarks to the EDMs of neutron and ${ }^{199} \mathrm{Hg}$ atom, we use the evaluation of the EDMs of neutron and ${ }^{199} \mathrm{Hg}$ atom in Ref. ${ }^{17}$,

$$
\begin{aligned}
d_{n} & =-1.6 \times e\left(d_{u}^{C}+0.81 \times d_{d}^{C}+0.16 \times d_{s}^{C}\right), \\
d_{\mathrm{Hg}} & =-8.7 \times 10^{-3} \times e\left(d_{u}^{C}-d_{d}^{C}+0.005 d_{s}^{C}\right),
\end{aligned}
$$

where $d_{n}$ is generated by the charged meson loops and $d_{\mathrm{Hg}}$ comes from the nuclear force by the pion exchange in the chiral perturbation theory. The experimental upperbounds on the EDMs of neutron ${ }^{18}$ and ${ }^{199} \mathrm{Hg}$ atom $^{19}$ are $\left|d_{n}\right|<6.3 \times 10^{-26} \mathrm{ecm}$ and $\left|d_{\mathrm{Hg}}\right|<1.9 \times 10^{-28} \mathrm{ecm}$, respectively (90\%C.L.). Thus, the upperbounds on the quark CEDMs are $e\left|d_{u}^{C}\right|<3.9(2.2) \times 10^{-26} e \mathrm{~cm}, e\left|d_{d}^{C}\right|<4.8(2.2) \times 10^{-26} \mathrm{e} \mathrm{cm}$, and $e\left|d_{s}^{C}\right|<2.4(44) \times 10^{-25} \mathrm{e} \mathrm{cm}$, from the EDM of neutron $\left({ }^{199} \mathrm{Hg}\right.$ atom $)$. Here, we assume that the accidental cancellation among the CEDMs does not suppress the EDMs. The constraint on $d_{s}^{C}$ from ${ }^{199} \mathrm{Hg}$ atom is one-

\footnotetext{
aThe up quark EDM and CEDM are also predicted in this model. Since they are suppressed by the bottom quark Yukawa coupling, they may be observable in near future when $\tan \beta$ is large ${ }^{15}$.
} 
order weaker than that from neutron, since the contribution to the EDM of ${ }^{199} \mathrm{Hg}$ atom is suppressed by $\pi^{0}-\eta^{0}$ mixing ${ }^{17}$.

In Fig. 2 the CEDMs for the down and strange quarks in the SUSY $\mathrm{SU}(5)$ GUT with the right-handed neutrinos are shown ${ }^{16}$. The minimal supergravity scenario is assumed and $M_{H_{c}}=2 \times 10^{16} \mathrm{GeV}$. In Fig. 2(a) the strange quark CEDM is presented as a function of the right-handed tau neutrino mass. We take $m_{\nu_{\tau}}=0.05 \mathrm{eV}$ and $U_{\mu 3}=1 / \sqrt{2}$. For the SUSY breaking parameters, $m_{0}=500 \mathrm{GeV}, A_{0}=0, m_{\tilde{g}}=500 \mathrm{GeV}$ and $\tan \beta=$ 10 , which lead to $\bar{m}_{\tilde{q}} \simeq 640 \mathrm{GeV}$. The contributions from the electron and muon neutrino Yukawa interactions to the flavor violation in the righthanded down-type squark mass matrix are ignored. The contributions are bounded by the constraints from the $K^{0}-\bar{K}^{0}$ mixing and $\operatorname{Br}(\mu \rightarrow e \gamma)$ when $\left|U_{e 2}\right| \sim 1 / \sqrt{2}$. From this figure, the right-handed tau neutrino mass should be smaller than $\sim 3 \times 10^{14} \mathrm{GeV}$. In Fig. 2(b), the down quark CEDM is presented as a function of the right-handed tau neutrino mass. This comes from non-vanishing $U_{e 3}$ in our assumption that the right-handed neutrino mass matrix is diagonal. The current bound is not significant even when $U_{e 3}=0.2$.
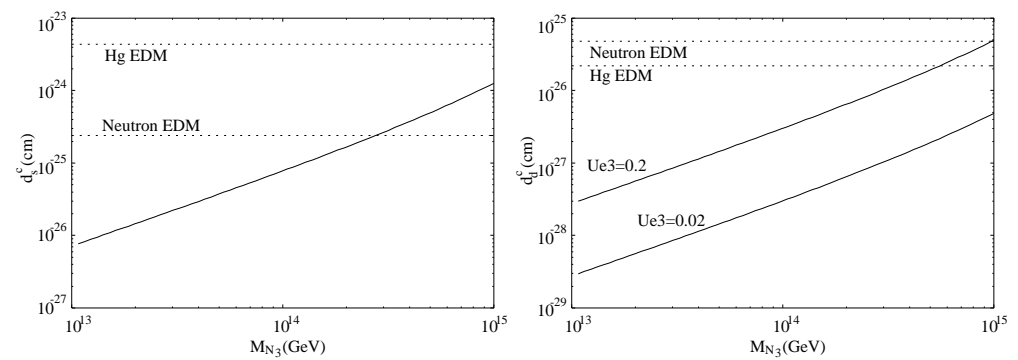

Figure 2. CEDMs for the strange quark in (a) and for the down quark in (b) as functions of the right-handed tau neutrino mass, $M_{N_{3}}$. Here, $M_{H_{c}}=2 \times 10^{16} \mathrm{GeV}, m_{\nu_{\tau}}=0.05 \mathrm{eV}$, $U_{\mu 3}=1 / \sqrt{2}$, and $U_{e 3}=0.2$ and 0.02 . For the MSSM parameters, we take $m_{0}=500 \mathrm{GeV}$, $A_{0}=0, m_{\tilde{g}}=500 \mathrm{GeV}$ and $\tan \beta=10$.

The new technique for the measurement of the deuteron EDM has a great impact on the quark CEDMs if it is realized ${ }^{20}$. If they establish the sensitivity of $d_{D} \sim 10^{-27} \mathrm{ecm}$, we may probe the new physics to the level of $e d_{s}^{C} \sim 10^{-26} e \mathrm{~cm}$ and $e d_{d}^{C} \sim e d_{u}^{C} \sim 10^{-28} \mathrm{e} \mathrm{cm}$, which are much stronger than the bounds from the neutron and ${ }^{199} \mathrm{Hg}$ atom EDMs ${ }^{17}$. This may imply that we may probe the structure in the neutrino sector even if $M_{N_{3}} \sim 10^{13} \mathrm{GeV}$ or $U_{e 3} \sim 0.02$. 
Finally, we discuss the correlation between the hadronic EDM and the $\mathrm{CP}$ asymmetry in $B \rightarrow \phi K_{s}\left(S_{\phi K_{s}}\right)$. The $b \rightarrow s \bar{s} s$ transition is sensitive to the new physics since it is induced at one-loop level ${ }^{21}$. When the righthanded down-type squarks have the flavor mixing, the $b-s$ penguin diagram may give a sizable contribution in $B \rightarrow \phi K_{s}$. Thus, if the deviation from the SM prediction in $B \rightarrow \phi K_{s}$ is observed, it might be a signature of the effect of the neutrino Yukawa coupling in the SUSY GUTs with the righthanded neutrinos. On the other hand, the strange quark CEDM is also generated in the model, and they have a strong correlation ${ }^{22}$.

The $b-s$ penguin contribution induced by the right-handed squark mixing is dominated by the gluino diagram, and it is represented by the effective operator $H=-C_{8}^{R}\left(g_{s} /\left(8 \pi^{2}\right)\right) m_{b} \overline{s_{R}}(G \sigma) b_{L}$. When both the left-handed and right-handed down-type squarks have flavor violation, we get a strong correlation between $C_{8}^{R}$ and $d_{s}^{C}$ as

$$
d_{s}^{C}=-\frac{m_{b}}{4 \pi^{2}} \frac{11}{21} \operatorname{Im}\left[\left(\delta_{L L}^{(d)}\right)_{23} C_{8}^{R}\right]
$$

up to the QCD correction. Here, we take $m_{\tilde{g}}=\bar{m}_{\tilde{d}}$. The coefficient $11 / 21$ in Eq. (13) changes from 1 to 1/3, depending on the SUSY mass spectrum.

In Fig. 3, the correlation between $d_{s}^{C}$ and $S_{\phi K_{s}}$ is shown, assuming Eq. (13). The detail of this figure is shown in Ref. ${ }^{17}$. Here, we take $\left(\Delta_{23}^{d}\right)_{L}=-0.04, \arg \left[C_{8}^{R}\right]=\pi / 2$ and $\left|C_{8}^{R}\right|$ corresponding to $10^{-5}<$ $\left|\left(\Delta_{32}^{d}\right)_{R}\right|<0.5$. $\kappa$ is a parameter for the matrix element of chromomagnetic moment in $B \rightarrow \phi K_{s}$, and we show the results for $\kappa=-1$ and -2 . From this figure, the deviation of $S_{\phi K_{s}}$ from the SM prediction due to the gluon penguin contribution should be suppressed when the constraints on $d_{s}^{C}$ from the ${ }^{199} \mathrm{Hg}$ atomic and the neutron EDMs are applied. We find that the neutron EDM gives a stronger bound on $S_{\phi K_{s}}$. Moreover, $S_{\phi K_{s}}$ may be constrained further by the future deuteron EDM measurements. Therefore, the hadronic EDMs give a very important implication to $S_{\phi K_{s}}$. Of cause, it should be careful to compare the EDM constraints with other low-energy observables, which are theoretically controlled better, since the hadronic EDMs may suffer from more hadronic uncertainties. However, the orders of the magnitude in the EDM constraints are still expected to have the significance.

\section{Summary}

In this article we review the CP violating phenomena in the SUSY seesaw models. In the SUSY extensions of the seesaw mechanism the neutrino 


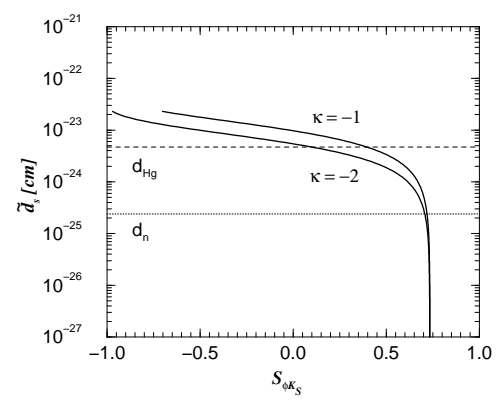

Figure 3. The correlation between $d_{s}^{C}$ and $S_{\phi K_{s}} . \kappa$ comes from the matrix element of chromomagnetic moment in $B \rightarrow \phi K_{s}$. The dashed (dotted) line is the upperbound on $d_{s}^{C}$ from the EDM of ${ }^{199} \mathrm{Hg}$ atom (neutron).

Yukawa interaction induces the flavor and $\mathrm{CP}$ violating sfermion mass terms via the radiative correction. Thus, we may probe the models by studying the flavor and $\mathrm{CP}$ violating phenomena.

The recent results for the $b$-s penguin processes, including $B \rightarrow \phi K_{s}$, in the BABAR and BELLE experiments are converging. The combined results of the various $b$-s penguin processes are $2.7 \sigma$ and $2.4 \sigma$ deviated from the $\mathrm{SM}$ prediction in the $\mathrm{BABAR}^{23}$ and BELLE ${ }^{24}$ experiments, respectively. Though it is premature to judge whether they are signals of new physics, it is important to discuss the sensitivity to new physics. The deviation may be explained by introduction of the right-handed bottom and strange squarks mixing, such as in the SUSY SU(5) GUT with the right-handed neutrinos, however, the hadronic EDM constraints give bounds on the deviation. Even if the discrepancy comes from the hadronic uncertainties, the further improvements of the bound on the hadronic EDMs and the $b$ - $s$ penguin processes are very important.

\section{References}

1. M. Gell-Mann, P. Ramond and R. Slansky, Proceedings of the Supergravity Stony Brook Workshop, New York, 1979, eds. P. Van Nieuwenhuizen and D. Freedman (North-Holland, Amsterdam); T. Yanagida, Proceedings of the Workshop on Unified Theories and Baryon Number in the Universe, Tsukuba, Japan 1979 (edited by A. Sawada and A. Sugamoto, KEK Report No. 79-18, Tsukuba).

2. M. Fukugita and T. Yanagida, Phys. Rev. D 42 (1990) 1285.

3. L. J. Hall, V. A. Kostelecky and S. Raby, Nucl. Phys. B 267 (1986) 415.

4. F. Borzumati and A. Masiero, Phys. Rev. Lett. 57 (1986) 961; J. Hisano, 
T. Moroi, K. Tobe, M. Yamaguchi and T. Yanagida, Phys. Lett. B 357 (1995) 579; J. Hisano, T. Moroi, K. Tobe and M. Yamaguchi, Phys. Rev. D 53 (1996) 2442; J. Hisano and D. Nomura, Phys. Rev. D 59 (1999) 116005.

5. Y. Farzan and M. E. Peskin, hep-ph/0405214.

6. J. R. Ellis, J. Hisano, M. Raidal and Y. Shimizu, Phys. Rev. D 66 (2002) 115013.

7. Y. Okada, K. Okumura and Y. Shimizu, Phys. Rev. D 58 (1998) 051901; Phys. Rev. D 61 (2000) 094001.

8. A. de Gouvea, S. Lola and K. Tobe, Phys. Rev. D 63 (2001) 035004.

9. J. R. Ellis, J. Hisano, S. Lola and M. Raidal, Nucl. Phys. B 621 (2002) 208.

10. J. R. Ellis, J. Hisano, M. Raidal and Y. Shimizu, Phys. Lett. B 528 (2002) 86.

11. For example, see J. Hisano and Y. Shimizu, Phys. Lett. B 565 (2003) 183; M. Ciuchini, A. Masiero, L. Silvestrini, S. K. Vempati and O. Vives, Phys. Rev. Lett. 92 (2004) 071801.

12. T. Moroi, Phys. Lett. B 493 (2000) 366.

13. S. Dimopoulos and L. J. Hall, Phys. Lett. B 344 (1995) 185.

14. R. Barbieri, L. J. Hall and A. Strumia, Nucl. Phys. B 445 (1995) 219; R. Barbieri, L. J. Hall and A. Strumia, Nucl. Phys. B 449 (1995) 437.

15. A. Romanino and A. Strumia, Nucl. Phys. B 490 (1997) 3.

16. J. Hisano, M. Kakizaki, M. Nagai and Y. Shimizu, hep-ph/0407169.

17. J. Hisano and Y. Shimizu, hep-ph/0406091.

18. P. G. Harris et al., Phys. Rev. Lett. 82 (1999) 904.

19. M. V. Romalis, W. C. Griffith and E. N. Fortson, Phys. Rev. Lett. 86 (2001) 2505.

20. Y. K. Semertzidis et al. [EDM Collaboration], AIP Conf. Proc. 698 (2004) 200.

21. Y. Grossman and M. P. Worah, Phys. Lett. B 395 (1997) 241; R. Barbieri and A. Strumia, Nucl. Phys. B 508 (1997) 3.

22. J. Hisano and Y. Shimizu, Phys. Lett. B 581 (2004) 224.

23. Talked by M. Giorgi in ICHEP'04, August 16-22, 2004 Beijing, China, (http://ichep04.ihep.ac.cn/).

24. Talked by Y. Sakai in ICHEP'04, August 16-22, 2004 Beijing, China, (http://ichep04.ihep.ac.cn/). 